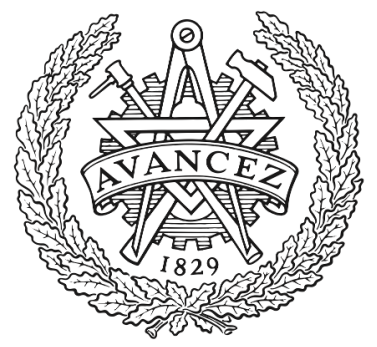

CHALMERS

UNIVERSITY OF TECHNOLOGY

\title{
Impact of Rough Surface Scattering on Stochastic Multipath Component Models
}

Downloaded from: https://research.chalmers.se, 2023-04-26 14:44 UTC

Citation for the original published paper (version of record):

Kulmer, J., Wen, F., Garcia, N. et al (2018). Impact of Rough Surface Scattering on Stochastic

Multipath Component Models. IEEE International Symposium on Personal, Indoor and Mobile Radio Communications, PIMRC: 1410-1416. http://dx.doi.org/10.1109/PIMRC.2018.8580964

N.B. When citing this work, cite the original published paper. 


\title{
Impact of Rough Surface Scattering on Stochastic Multipath Component Models
}

\author{
Josef Kulmer*, Fuxi Wen ${ }^{\dagger}$, Nil Garcia ${ }^{\dagger}$, Henk Wymeersch ${ }^{\dagger}$, Klaus Witrisal* \\ ${ }^{*}$ Graz University of Technology, Austria; ${ }^{\dagger}$ Chalmers University of Technology, Sweden \\ email: kulmer@tugraz.at
}

\begin{abstract}
Multipath-assisted positioning makes use of specular multipath components (MPCs), whose parameters are geometrically related to the positions of the transceiver nodes. Diffuse scattering from rough surfaces affects the observed specular reflections in the angular and delay domains. Based on the effective roughness approach, the angular delay power spectrum can be calculated as a function of location parameters, which-in a next step-could be useful to accurately characterize the position-related information of MPCs. The calculated power spectra follow reported characteristics of stochastic multipath models, i.e. Gaussian shape in the angular domain and an exponential shape in the delay domain. The resulting angular and delay spreads are in an equivalent range to values reported in literature.
\end{abstract}

\section{INTRODUCTION}

Recent work in radio-based positioning exploits position-related information of multipath propagation [1], [2]. For this purpose, the multipath propagation is described using geometry-based stochastic channel models. These channel models characterize positionrelated information using geometrical relations between transmitter and receiver as well as the surrounding reflective objects. Geometrical optics then allows a proper modeling of specular multipath components (MPCs).

The stochastic channel component describes dense multipath, stemming from interaction with rough surfaces or small objects. In the context of localization, attempts are taken for a stochastic description of dense multipath, i.e. its characterization as zero-mean additive white Gaussian noise [2], or by placing additional scatter points [3] in the geometric setup. However, the characterization of the stochastic channel component will affect the potential outcome of localization algorithms. Proper stochastic models of dense multipath are required.

In literature, (geometry-based) stochastic channel models are parameterized by the standards IEEE 802.15.3c [4], IEEE 802.11ad [5] or 3GPP TR38.901 [6]. The purely stochastic channel models IEEE 802.15.3c [4] and 3GPP TR38.901 [6] rely on the Saleh Valenzuela propagation model which assumes that radio frequency power arrives in clusters [7]. The

This work was supported by European Commission H2020-MSCAIF-2015 project No.700044 (MASSLOC) and H2020-ICT-2016-2 project No.761510 (5GCAR) as well as COST ACTION CA15104 IRACON.
MPCs within a cluster are described statistically using empirical distributions. The parameters of the empirical distributions are fitted independently in the angular and delay domains. Both IEEE 802.15.3c and 3GPP TR38.901 have in common that the cluster parameters are not identified as function of a geometry based setup of transmitter / receiver.

The geometry-based stochastic channel model IEEE $802.11 \mathrm{ad}$ [5] aims at relating cluster parameters to a location specific setup. A main component within each cluster is identified, based on ray tracing, which can be classified as a specular component originating from a reflective surface, as illustrated in Figure 1. The main component is accompanied by dense multipath components stemming from a rough surface or small objects in its vicinity. These dense MPCs are unpredictable by ray tracing and parameterized in a stochastic manner using empirical distributions [5], [8], similarly to [4], [6].

The stochastic descriptions of dense multipath cover general scenarios, characterizing the average scattering behavior of reflective materials. Material specific scattering can be elaborated using additional information, i.e. the material's geometric dimension and dielectric parameters or both. In [9], [10] knowledge of material properties enable the prediction of the electromagnetic field. A stochastic description of rough surfaces is employed in [11]-[13] where the height of the rough surface is assumed to follow a zero-mean Gaussian density. Motivated by radiation theory, the authors in [14], [15] assume a certain scattering lobe for an incident ray, denoted as effective roughness approach. This approach is successfully employed in ray tracing [16]-[18], point cloud scattering [19]-[23] or propagation graph theory [24], [25]. It has been parameterized for several materials [23], [26]. Although effective roughness has shown to produce proper scattering models, its consequences for the channel's angular delay power spectrum (ADPS) are not discussed adequately.

In this paper we discuss the stochastic nature of dense multipath stemming from rough surfaces, e.g. rough cast as well as balconies. Based on the effective roughness approach, we propose a scattering function which is symmetric with respect to transmitter and receiver positions. The scattering function enables the calculation of a 


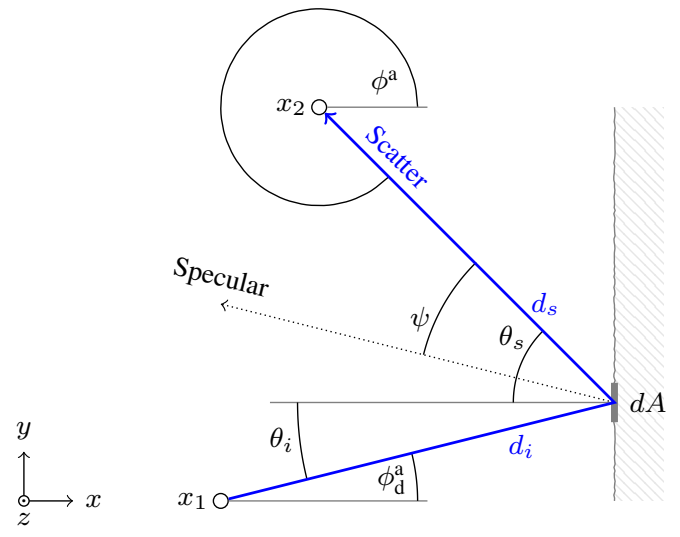

Fig. 1. Illustration of a reflective surface, located along the $y z$ plane, and the path from transceiver 1 , located at $x_{1}$, to transceiver 2 at $x_{2}$ via surface area element $d A$.

joint angular delay power spectrum. The power spectrum describes dense multipath in a stochastic manner as a function of the geometric setup and the parameters of the rough surface. We analyze the power spectrum in the angular and delay domains and compare our results with measurements reported in literature.

The importance of this work for multipath-assisted positioning is as follows: Results in [27], [28] demonstrate that the range-information contributed by each MPC is related to the power ratio of the specular component and the dense multipath interfering with it, the so-called signal-to-interference-plus-noise ratio (SINR). The ADPS is needed to compute the SINR for some given probing signal with a defined bandwidth and directivity of the transceiver antennas. The SINR also agrees with the Ricean K-factor characterizing the amplitude fading of the MPC. The coherence distance of this fading is known to be related to the angular power spectrum. We therefore expect the results of this paper to be useful for the derivation of position error bounds and position estimation algorithms under realistic channel conditions.

\section{SCATTERING FUNCTION AND IMPLICATIONS ON THE ANGULAR DELAY POWER SPECTRUM}

We aim at relating the observed angular delay power spectrum to parameters based on the geometric setup using scattering models, which are originally introduced in [14], [15]. The scattering model treats a single planar, reflective surface which is illuminated by a transmitter. The impinging power at the reflective surface is attenuated and subsequently scattered in various directions. Parts of the reflected power arrive at the receiver with a certain ADPS. Figure 1 illustrates the setup for the azimuth domain.

\section{A. Scattering model}

Based on the Radar equation [29], the Lambertian [14] and directional scattering [15], we model the differential received power $d P_{r}$ observed from differential surface element $d A$ according to

$$
d P_{r}=\frac{P_{t} \cos \theta_{i}}{d_{i}^{2}} \times \frac{d A \cos \theta_{s}}{d_{s}^{2}} \times \frac{(1+\cos \psi)^{\alpha_{R}}}{k_{\alpha_{R}} 2^{\alpha_{R}}}
$$

where we set both effective aperture area of the receiving antenna and the antenna gain of the transmitting antenna to 1 . The first factor on the right-hand-side defines the intensity at surface area element $d A$ with distance $d_{i}$ (see Fig. 1) and transmitted power $P_{t}$. The angle $\theta_{i}$ between impinging ray and surface normal attenuates the intensity at $d A$ according to the cosine law. Multiplication with area $d A$ yields the reflected power which is scattered towards various directions. The second factor defines the recognized intensity by the receiver, scaled by the squared distance $d_{s}^{2}$, where the cosine law is addressed by the angle $\theta_{s}$ between the scattered path and the surface normal.

The third factor determines the impact from directive scattering, motivated by [15], where we assume that the majority of the impinging power is scattered along the direction of the specular one. Deviating scatter angles, denoted by angle $\psi$, lower the received power. The parameter $\alpha_{R}$ controls the level of surface roughness. At walls with only small levels of roughness (in comparison to the wavelength of mmWaves, e.g. window glass) the scattering lobe is narrow, controlled by high levels of $\alpha_{R}$. Increasing levels of roughness yield spreading in various directions, described by small levels of $\alpha_{R}$. The remaining $k_{\alpha_{R}}$ is a scaling factor such that the scattered power is independent on $\alpha_{R}$ and results by integration of factor 3 over $\psi$. The scattering attenuation factor, modeled in [14], [15], is neglected in (1) as it has no impact on the evaluated angular and delay spreads.

The presented scattering model is strongly related to the Lambertian [14] and the single-lobe directional scattering model [15]. It enables a smooth transition from Lambertian scattering (setting $\alpha_{R}=0$ ) to directional scattering $\left(\alpha_{R} \rightarrow \infty\right)$. Note that the single-lobe scattering model [15] treats the impinging wave using the cosine law, and the scattered wave by a directional scattering lobe. Hence, in a non-symmetric setup of transmitter / receiver position, the outcome will differ if the transceivers change their roles. The scattering model presented in (1) preserves symmetry where we assume that both impinging and scattered waves are attenuated by the cosine law plus an additional scattering lobe.

Figure 2 illustrates the scattering power for various directions $\theta_{s}$ of the Lambertian - [14], directional -..... [15, single-lobe directional model] and the proposed scattering model - for an impinging angle $\theta_{i}=30^{\circ}$ with $\alpha_{R}=2$. We can observe that at Lambertian 


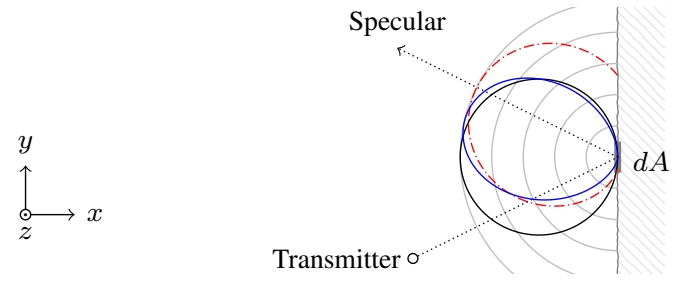

Fig. 2. Polar plot of scattered power at $d A$ for an impinging MPC with $\theta_{i}=30^{\circ}$ illustrating the Lambertian —_ directional _..... and proposed model $\longrightarrow$. The dotted arrow is the specular component.

scattering the impinging power is scattered according to the cosine law. The directional as well as the proposed scattering model radiate its power along the specular component.

The scattering functions describe the received differential power from a specific differential area element $d A$. In the following we rephrase (1) in order to calculate the resulting ADPS as observed by the receiver.

\section{B. Derivation of angular delay power spectrum}

Having the differential scattering power $d P_{r}$ from differential area $d A$ we aim at calculating the joint ADPS. The power spectrum describes the spreading of the received power along the angular and delay domains. Integration of the ADPS over $\tau$ results in the angular power spectrum (APS). Equivalently, the delay power spectrum (DPS) is established by integration of the ADPS over both azimuth and elevation angles. Note, (1) is symmetric with respect to the position of transmitter and receiver and thus, the observed DPS remains equal if the transceivers exchange their role of transmitting and receiving. Still, in general the APS is different, i.e. the APS at position $x_{2}$ for a transmitting node at position $x_{1}$ is different to the APS at $x_{1}$ for a transmitting node at $x_{2}$.

1) Joint angular delay power spectrum: The scattering model in (1) describes the received power from a desired area. In order to calculate the joint ADPS, we express the desired area as a function of azimuth $\phi^{\mathrm{a}}$, elevation $\phi^{\mathrm{e}}$ and delay $\tau$. Assuming the reflective surface is aligned with the $y z$-plane, we can substitute the differential area $d A=d \Omega d_{s}^{2} /\left(\cos \phi^{\mathrm{a}} \cos \phi^{\mathrm{e}}\right)$ by a solid angle $d \Omega$ [30] which can be subsequently described as $d \Omega=\cos \phi^{\mathrm{e}} d \phi^{\mathrm{a}} d \phi^{\mathrm{e}}$ of azimuth $\phi^{\mathrm{a}}$ and elevation angle $\phi^{\mathrm{e}}$, resulting in $d A=d_{s}^{2} /\left(\cos \phi^{\mathrm{a}}\right) d \phi^{\mathrm{a}} d \phi^{\mathrm{e}}$. Substituting $d A$ in (1) enables to calculate the total received power $P_{r}=\int d P_{r}$ and the ratio $P_{r} / P_{t}$,

$$
\frac{P_{r}}{P_{t}}=\iiint p\left(\phi^{\mathrm{a}}, \phi^{\mathrm{e}}, \tau\right) d \phi^{\mathrm{a}} d \phi^{\mathrm{e}} d \tau
$$

where $p\left(\phi^{\mathrm{a}}, \phi^{\mathrm{e}}, \tau\right)$ denotes the spreading of the received power in the angular and delay domains, defined as joint
ADPS

$$
\begin{aligned}
& p\left(\phi^{\mathrm{a}}, \phi^{\mathrm{e}}, \tau\right) \triangleq \\
& \quad \frac{\cos \theta_{i} \cos \theta_{s}}{k_{\alpha_{R}} d_{i}^{2} \cos \phi^{\mathrm{a}}} \frac{(1+\cos \psi)^{\alpha_{R}}}{2^{\alpha_{R}}} \delta\left(\tau-f_{\tau}\left(\phi^{\mathrm{a}}, \phi^{\mathrm{e}}\right)\right) .
\end{aligned}
$$

The Dirac delta $\delta(\cdot)$ relates a pair of angles $\left(\phi^{\mathrm{a}}, \phi^{\mathrm{e}}\right)$ to its corresponding delay $\tau$ where the function $\tau=$ $f_{\tau}\left(\phi^{\mathrm{a}}, \phi^{\mathrm{e}}\right)$ is calculated using trigonometric identities. The location dependent parameters $\left\{\theta_{i}, \theta_{s}, d_{i}, \psi\right\}$ in (2) are also derived from $\left(\phi^{\mathrm{a}}, \phi^{\mathrm{e}}\right)$.

2) Angular power spectrum: The azimuth APS $p\left(\phi^{\mathrm{a}}\right)$ is calculated by integration of (2) along is elevation and delay domains, according to

$$
p\left(\phi^{\mathrm{a}}\right)=\iint p\left(\phi^{\mathrm{a}}, \phi^{\mathrm{e}}, \tau\right) d \phi^{\mathrm{e}} d \tau .
$$

Analytic solutions to (3) are challenging due to nonlinear relations between angles and geometric setup between both transceivers. We propose a numeric approximation by discretizing the angles. Therefore, we generate $N_{\phi}$ uniformly distributed angle pairs $\left\{\left(\phi_{i}^{\mathrm{a}}, \phi_{i}^{\mathrm{e}}\right)\right\}_{i=1}^{N_{\phi}}$ where each pair $\left(\phi_{i}^{\mathrm{a}}, \phi_{i}^{\mathrm{e}}\right)$ has a constant solid angle $\Delta \Omega=$ $4 \pi / N_{\phi}$. Then, (3) can be written as

$$
p\left(\phi^{\mathrm{a}}\right) \approx \sum_{\phi^{\mathrm{e}} \in \mathcal{P}_{\phi^{\mathrm{a}}}} p\left(\phi^{\mathrm{a}}, \phi^{\mathrm{e}}, \tau\right) \frac{\Delta \Omega}{\Delta \phi^{\mathrm{a}}}
$$

where $\mathcal{P}_{\bar{\phi}^{\mathrm{a}}}=\left\{\phi_{i}^{\mathrm{e}}: 0 \leq\left|\bar{\phi}^{\mathrm{a}}-\phi_{i}^{\mathrm{a}}\right|<\Delta \phi^{\mathrm{a}} / 2\right\}$ contains the elevation angles $\left\{\phi_{i}^{\mathrm{e}}\right\}$ associated to the azimuth angle of interest $\bar{\phi}^{\mathrm{a}}$, with desired resolution $\Delta \phi^{\mathrm{a}}$. The summation along the elevation angles for a desired azimuth angle accounts for both integrals in (3) where we apply the Dirac delta's sifting property.

The elevation APS follows equivalently to (3) by integration along the azimuth domain.

3) Delay power spectrum: Finally we calculate the DPS by integration of the joint ADPS (2) along both angles, according to

$$
p(\tau)=\iint p\left(\phi^{\mathrm{a}}, \phi^{\mathrm{e}}, \tau\right) d \phi^{\mathrm{a}} d \phi^{\mathrm{e}} .
$$

Here, the DPS for a desired delay $\bar{\tau}$ results by integration along angles $\left(\phi^{\mathrm{a}}, \phi^{\mathrm{e}}\right)$ which fulfill $\bar{\tau}=f_{\tau}\left(\phi^{\mathrm{a}}, \phi^{\mathrm{e}}\right)$. Thus, we can approximate (5) as sum along angle pairs $\left(\phi^{\mathrm{a}}, \phi^{\mathrm{e}}\right) \in \mathcal{T}_{\bar{\tau}}$ with $\mathcal{T}_{\bar{\tau}}=\left\{\left(\phi_{i}^{\mathrm{a}}, \phi_{i}^{\mathrm{e}}\right): 0 \leq \mid \bar{\tau}-\right.$ $\left.f_{\tau}\left(\phi_{i}^{\mathrm{a}}, \phi_{i}^{\mathrm{e}}\right) \mid<\Delta \tau / 2\right\}$, whose corresponding delay $\tau$ is equal to the desired delay $\bar{\tau}$, up to numeric resolution $\Delta \tau$, according to

$$
p(\tau)=\sum_{\left(\phi^{\mathrm{a}}, \phi^{\mathrm{e}}\right) \in \mathcal{T}_{\tau}} p\left(\phi^{\mathrm{a}}, \phi^{\mathrm{e}}\right) \frac{\Delta \Omega}{\Delta \tau} .
$$

In the following we compare the proposed scattering model and the presented ones in [14], [15] in terms of resulting APS and DPS. 


\section{Analysis and comparison of APS}

Application of the scattering models enables the calculation of a theoretic APS to be expected at the transceiver positions. In this section we aim at comparing the proposed scattering model with [14] (denoted as Lambertian) and [15] (denoted as directional scattering). We evaluate the azimuth's APS for two transceivers and three different levels of roughness, determined by $\alpha_{R} \in\{0,2,10\}$. We consider two scenarios of positions of transceivers. Scenario (a) examines a symmetric setup where the surface is located along the $y z$ plane at $x=0$, transceiver $x_{1}$ at $(x, y, z)$ coordinates of $x_{1}=$ $(-10,-5,0)$ and transceiver $x_{2}$ at $x_{2}=(-10,5,0)$, as illustrated in Fig. 3. Scenario (b) shows a nonsymmetric setup where transceiver $x_{2}$ moves to position $x_{2}=(-5,5,0)$ and the transceiver $x_{1}$ persists at $x_{1}=$ $(-10,-5,0)$ (Fig. 4). In the numerical approximations we set $N_{\phi}=10^{6}, \Delta \phi^{\mathrm{a}}=\pi / 100 \mathrm{rad}$ and $\Delta \tau=1 \mathrm{~ns}$.

1) Symmetric scenario: Starting with the symmetric scenario (a), Figure 3 illustrates the APS of the proposed scattering model for various values of $\alpha_{R}=0$-, $\alpha_{R}=2-$ and $\alpha_{R}=10-$. We compare the outcome to the Lambertian - (mathematically identical to the proposed one with $\alpha_{R}=0$ ), and to the directional model using $\alpha_{R}=2-\cdots$ and $\alpha_{R}=10$----. The calculation shows the azimuth plane where we set the elevation to $\phi^{\mathrm{e}}=0$. The direction of the specular component between both transceivers is shown as dotted, gray line. The APS of $x_{1}\left(x_{2}\right)$ are calculated assuming $x_{2}\left(x_{1}\right)$ radiates in an isotropic manner.

We can observe that the patterns are symmetric whether $x_{1}$ transmits to $x_{2}$ or opposite. A comparison between directional and proposed scattering model shows strong similarities. Note that, the directional model spreads its peak power along the specular component (as illustrated in Figure 2) but the peak power at the receiving node $\left(x_{1}\right.$ or $\left.x_{2}\right)$ is not received from the direction of the specular component. This is a crucial finding and can be explained by the fact that the level of received scattering power from area $d A$ depends on the illuminated intensity at $d A$. The intensity is largest in the vicinity of the transmitter with the consequence of increased scatter power from surface areas, close to the transmitter. At $\alpha_{R}=0$ the peak power is received from a scattering point with shortest distance between transmitter and reflective surface. An increasing level of $\alpha_{R}$ narrows the angular spread. The angle's peak power moves towards the angle of the specular component.

2) Non-symmetric scenario: We proceed by evaluating the APS for the non-symmetric scenario (b) where $x_{2}$ is aligned closer to the reflective surface than $x_{1}$ yielding non-symmetric APS as well. This can be argued since $x_{2}$ is aligned close to the reflective surface while the distance between $x_{1}$ and surface area elements in the vicinity of $x_{1}$ is large. Thus, these surface area elements
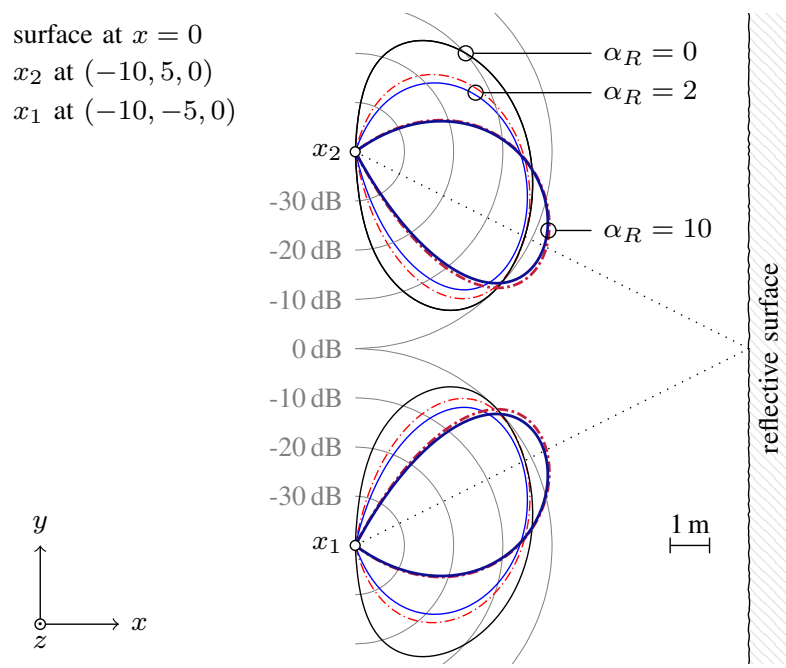

Fig. 3. Polar plot of APS of the symmetric scenario using the proposed scattering model with $\alpha_{R}=0 \longrightarrow, \alpha_{R}=2 \longrightarrow$ and $\alpha_{R}=10$ — in comparison to Lambertian — (equivalent to the proposed one with $\alpha_{R}=0$ ) and directional one, using $\alpha_{R}=2$-.... and $\alpha_{R}=10-\cdot-\cdot$.
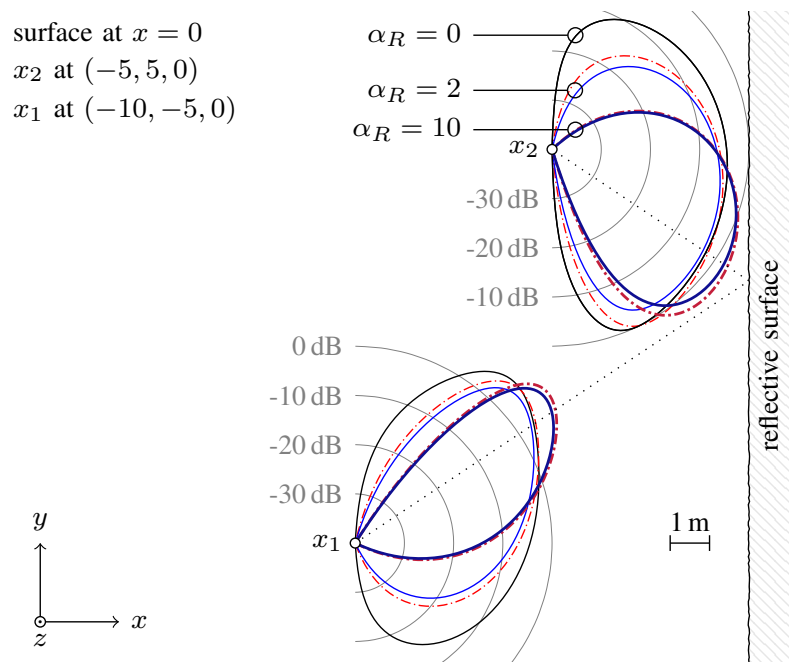

Fig. 4. Equivalent to Figure 3 with $x_{2}$ at $(-5,5,0)$.

are illuminated by a similar power which is scattered to $x_{2}$. The receiver observes arriving power from a wide range of directions which results in an increased angular spread.

Equivalent to the observations made at the symmetric scenario, the angular spread of the Lambertian scattering is largest. The angle's peak power moves towards the specular component's angle for rising $\alpha_{R}$. Furthermore, the angular spread is reduced for increased $\alpha_{R}$.

\section{Analysis and comparison of the DPS}

Finally, in Figure 5 we illustrate the delay power spectra for the symmetric and non-symmetric scenarios. In general, the magnitude scaling changes drastically showing a strong sensitivity to the delay domain. We 

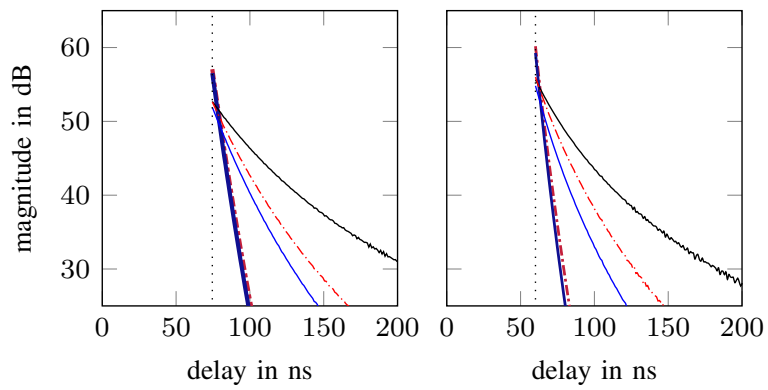

Fig. 5. Delay power spectrum of scenario (a) (left) and (b) (right) using $\alpha_{R}=0 \longleftarrow, \alpha_{R}=2 \longrightarrow$ and $\alpha_{R}=10-$ in comparison to Lambertian — , and the directional model using $\alpha_{R}=2 \ldots$ and $\alpha_{R}=10-\cdot-\cdot-$. The specular component's delay is shown as dotted, vertical line.

can observe that the peak power arrives simultaneously with the specular component's delay (black dotted). At increasing delays the power is attenuated. The lowest power decay rate is achieved by the Lambertian scattering. Rising $\alpha_{R}$ (equivalent to reduced roughness) increases the power decay rate. This can be argued since the directional models reward dense multipaths stemming from the direction of the specular components.

We can conclude that the proposed scattering function is identical to the Lambertian model by setting $\alpha_{R}=0$. At increased $\alpha_{R}$ it converges to the outcome of the directional scattering model, with the advantage of preserving a symmetric channel between both transceivers. Hence, in the following we proceed with the proposed scattering function and compare its shape with empirical distributions from literature.

\section{RELATION OF SCATTERING MODEL TO EMPIRICAL DISTRIBUTIONS}

The analysis and comparison of the proposed scattering model showed its potential to model both Lambertian and directional scattering. In this section we aim at relating the scattering model to empirical distributions, identified in literature and compare the calculated angular and delay spread to reported ones.

\section{A. Empirical distribution of MPCs in literature}

Empirical distributions of MPCs are provided by standardized channel models IEEE 802.15.3c [4], IEEE 802.11.ad [5] or 3GPP TR38.901 [6]. In these channel models, estimated MPCs are associated to clusters in angular delay domain using visual inspection or machine supported clustering [31]. Considering a single cluster, in angular domain, a (wrapped) Gaussian distribution [4], [6] or Laplacian [4], [32] with standard deviation $\sigma_{\phi^{\mathrm{a}}}$ was identified for the azimuth, and a Laplacian with standard deviation $\sigma_{\phi^{e}}$ for the elevation angle. The mean value is calculated as average angle of the MPCs within a cluster. In delay domain, the MPCs follow an exponential decay [4], [6] with rate $\gamma$, related to
TABLE I

IDENTIFIED RANGES OF PARAMETERS FOR THE REPORTED SCENARIOS OF IEEE 802.15.3C, IEEE 802.11 AD, 3GPP TR38.901 AND [32] WHERE (L), (G), (WG) DENOTE LAPLACIAN, GAUSSIAN AND WRAPPED GAUSSIAN DISTRIBUTION.

\begin{tabular}{|c|c|c|c|c|}
\hline & IEEE & IEEE & $3 \mathrm{GPP}$ & [32] \\
\hline & $802.15 .3 \mathrm{c}$ & $802.11 \mathrm{ad}$ & TR38.901 & \\
\hline$\sigma_{\phi^{\mathrm{a}}}$ in ${ }^{\circ}$ & 10 & $5-10(\mathrm{G})$ & $2-22(\mathrm{WG})$ & $17-40(\mathrm{~L})$ \\
\hline$\sigma_{\phi^{\mathrm{e}}}$ in ${ }^{\circ}$ & - & $5-10(\mathrm{G})$ & 3-9 (L) & $11-17(\mathrm{~L})$ \\
\hline$\alpha$ in $\mathrm{ns}$ & 7 & $4.5-8.7$ & $5-11$ & $4.6-4.8$ \\
\hline
\end{tabular}

the cluster's first path. It is interesting to note that [5] differs to [4], [6] by concatenating a rising and falling (pre and post cursor) exponential decay. Furthermore, in [5] a cluster-specific K-factor is defined as power ratio between a main component and the residual cluster paths. Hence, a comparison between the standardized channel models is not straight forward due to different angular distributions ((wrapped) Gaussian or Laplacian) and delay distributions (single decay and double decay).

Table I presents the identified parameters from [4], [5, post cursor decay rate], [6], [32]. We can observe a wide range of the azimuth standard deviation $\sigma_{\phi^{a}}$ but narrow elevation $\sigma_{\phi^{e}}$. The decay rates are in a similar range in low ns ranges of 4 to $11 \mathrm{~ns}$.

\section{B. Evaluation in angular domain}

In angular domain, (wrapped) Gaussian or Laplacian or both distributions have been proposed to model the APS. Both Gaussian and Laplacian distribution are parameterized by a mean angle $\bar{\phi}$ and standard deviation $\sigma_{\phi}$. In literature, $\bar{\phi}$ and $\sigma_{\phi}$ are calculated as first and central second moments (equivalent to the definitions of the mean and rms angular spreads). Figure 6 illustrates the APS at $x_{1}$ along the azimuth angle for scenario (b) for two levels of $\alpha_{R}=0-$ and $\alpha_{R}=10$ - The Gaussian distribution (dashed) is shown for comparison. We can observe that at $\alpha_{R}=0$ the Gaussian approximation is not able to follow the derived APS. At increasing $\alpha_{R}=10$ the power is centralized along its mean value, thus, a Gaussian approximation is more appropriate.

We have repeated the calculation of APS for both scenarios. Table II reports the identified rms angular spreads as well as the gap $\epsilon_{\bar{\phi}}$ between the angles of the specular component and the mean angle from the APS. In case of the symmetric scenario (a) the APS as seen from transceiver $x_{1}$ is identical to the APS as seen from $x_{2}$. At the non-symmetric scenario (b) $x_{2}$ is located closer to the reflective surface, yielding increased angular spread in comparison to $x_{1}$. Decreasing levels of roughness (equivalent to narrowing the scattering lobe) from $\alpha_{R}=0$ to $\alpha_{R}=10$ lower the angular spread from approximately $31^{\circ}$ to $12^{\circ}$ in average. The angle gap $\epsilon_{\bar{\phi}}$ is largest at high levels of roughness and gets reduced with increasing $\alpha_{R}$. Moreover, in non-symmetric setup (b) 


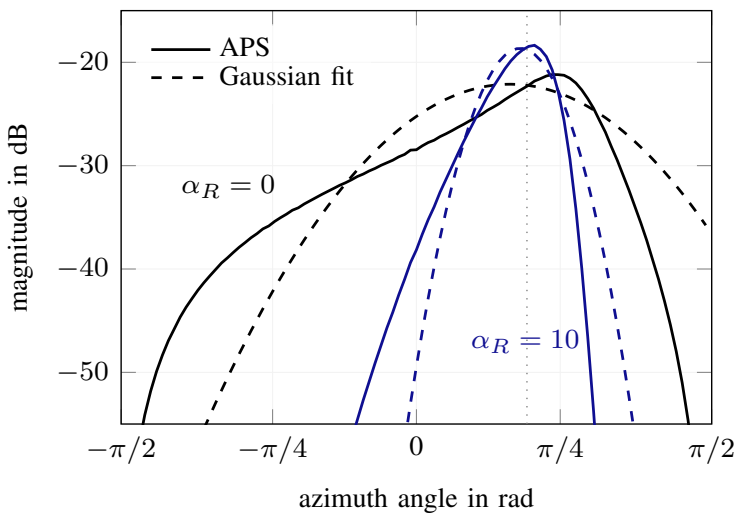

Fig. 6. Illustration of APS at $x_{1}$ using scenario (b) with high levels $\alpha_{R}=0$ (black) and low levels of roughness $\alpha_{R}=10$ (blue).

TABLE II

IDENTIFIED AZIMUTH ANGULAR SPREAD FOR VARIOUS LEVELS OF ROUGHNESS $\alpha_{R}$ FOR BOTH SCENARIOS (A) AND (B).

\begin{tabular}{rrrrrrrr} 
& $\alpha_{R}$ & 0 & 2 & 4 & 6 & 8 & 10 \\
\hline \hline (a) & $\sigma_{\phi^{\mathrm{a}}}, \sigma_{\phi_{\mathrm{d}}^{\mathrm{a}}}$ in ${ }^{\circ}$ & 32.2 & 21.6 & 17.2 & 14.7 & 13.1 & 11.9 \\
& $\epsilon_{\bar{\phi}}$ in ${ }^{\circ}$ & 9.4 & 4.7 & 3.0 & 2.1 & 1.5 & 1.2 \\
\hline \hline (b) & $\sigma_{\phi_{\mathrm{d}}^{\mathrm{a}}}$ in ${ }^{\circ}$ at $x_{1}$ & 23.8 & 15.7 & 12.4 & 10.5 & 9.3 & 8.4 \\
& $\epsilon_{\bar{\phi}}$ in ${ }^{\circ}$ at $x_{1}$ & 5.2 & 3.5 & 2.9 & 2.5 & 2.2 & 2.0 \\
\hline (b) & $\sigma_{\phi^{\mathrm{a}}}$ in ${ }^{\circ}$ at $x_{2}$ & 36.8 & 26.3 & 21.5 & 18.6 & 16.7 & 15.3 \\
& $\epsilon_{\bar{\phi}}$ in ${ }^{\circ}$ at $x_{2}$ & 10.4 & 4.9 & 2.9 & 1.8 & 1.2 & 0.8 \\
\hline \hline
\end{tabular}

$\epsilon_{\bar{\phi}}$ is increased if the transceiver is located close to the reflective surface.

\section{Evaluation in delay domain}

In delay domain, IEEE 802.15.3c and 3GPP TR38.901 have identified an exponential decay, parameterized by decay rate $\gamma$. We calculate the decay rate as first moment of the DPS with respect to the excess array. Figure 7 illustrates the DPS of the non-symmetric scenario using the proposed scattering model. The fitted exponential decay function (dashed) is shown in comparison. We have evaluated $\gamma$ for both scenarios as function of $\alpha_{R}$, as shown in Table III. At high levels of roughness $\left(\alpha_{R}=0\right)$ the arriving power is spread in the delay domain, resulting in an average decay rate of $\gamma \approx 16 \mathrm{~ns}$ (across both scenarios). Rising levels of $\alpha_{R}$ yield decreasing $\gamma \approx 2.2 \mathrm{~ns}$.

\section{Discussion}

The calculated angular power spectrum show a bias between the specular component's angle and the mean value of the dense multipath power. Furthermore, the

TABLE III

IDENTIFIED DECAY RATES OF THE DPS FOR VARIOUS LEVELS OF ROUGHNESS $\alpha_{R}$ FOR BOTH SCENARIOS (A) AND (B).

\begin{tabular}{llllllll} 
& $\alpha_{R}$ & 0 & 2 & 4 & 6 & 8 & 10 \\
\hline Scen. (a) & $\gamma$ in ns & 14.3 & 6.1 & 3.9 & 2.9 & 2.3 & 1.9 \\
Scen. (b) & $\gamma$ in ns & 17.8 & 7.6 & 4.8 & 3.5 & 2.8 & 2.3 \\
\hline
\end{tabular}

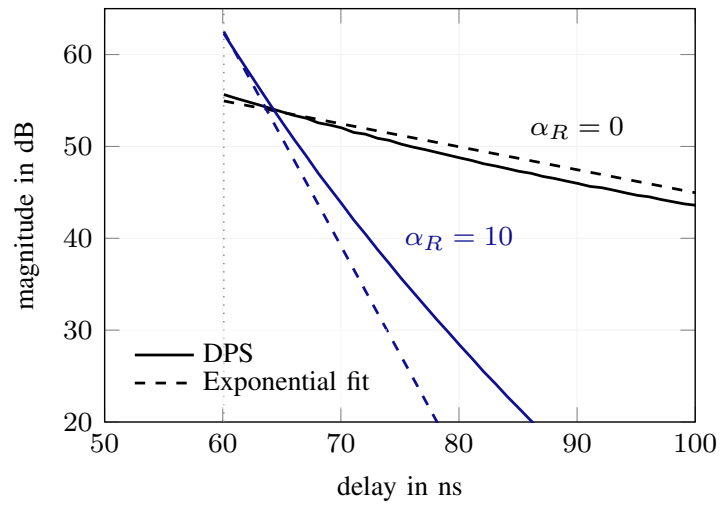

Fig. 7. Illustration of DPS using scenario (b) with high levels $\alpha_{R}=0$ (black) and low levels of roughness $\alpha_{R}=10$ (blue).

APS are not symmetric along their mean value. These findings encourage the consideration of dense multipath statistics for an unbiased estimation of the specular component's angle.

A comparison between the reported parameters from Table I and the calculated ones in Table II and III show strong accordance and both angular and delay parameters are in a similar range. Still, the parameters are calculated for an infinite dimension of a planar surface. Limiting dimensions yield narrower APS as well as decreased decay rates. On the other hand, strong surface variations (like balconies or other heavily structured surfaces) may increase the decay rates which limits the explanatory power of this comparison. Furthermore, the results differ as function of the considered scenario, showing a strong dependency on the location setup. However, based on our findings, we support the average value of $\alpha_{R}=4$, as proposed in [15].

The calculated shape of the DPS fits well to the reported exponential decay from IEEE 802.15.3c and 3GPP TR38.901. The double exponential decay, identified in IEEE 802.11ad, cannot be verified. This may be explained since the scattering model does not explicitly incorporate variations in the surface's heights or objects in the vicinity of the surface. Furthermore, the cluster's main component, identified by IEEE 802.11ad, is not predicted by the scattering model. It is worth noting that ray tracing applications consider an additional specular component [16] which may act as cluster's main MPC.

Our calculations consider isotropic radiating antennas while at mmWaves, reported cluster parameters are often based on measurements with highly directive horn antennas. A narrow antenna's radiation pattern will affect the angular cluster spread which lowers the explanatory power from the comparison with isotropic antennas. However, our literature study did not show a clear relation between radiation pattern and angular spread, i.e. narrower radiation pattern does not necessarily result in narrower angular spreads [33]. These findings are 
supported by preliminary evaluations of (2) where we considered the antenna's directivity in the angular domain. The resulting angular spread value can get raised or reduced, depending on the combination of geometric setup and antenna directivity.

\section{CONCLUSiON}

We have proposed a symmetric scattering function, capable of modeling both Lambertian and directional scattering. The scattering function enables to derive the APS and DPS as functions of the geometric setup. The APS shows that the majority of arriving dense multipath power deviates from the direction of the specular component. Both APS and DPS are in accordance to reported empirical distributions and fitted parameters. In context of localization, dense multipath from rough surfaces adds non-zero-mean noise to specular components resulting in biased angular and delay estimates if not corrected for.

\section{ACKNOWLEDGMENT}

We are grateful to Dr. V. Degli-Esposti for the constructive discussions throughout this research.

\section{REFERENCES}

[1] K. Witrisal, P. Meissner, E. Leitinger, Y. Shen, C. Gustafson, F. Tufvesson, K. Haneda, D. Dardari, A. F. Molisch, A. Conti, and M. Z. Win, "High-accuracy localization for assisted living," IEEE Signal Process. Mag., 2016.

[2] A. Shahmansoori, G. E. Garcia, G. Destino, G. Seco-Granados, and $\mathrm{H}$. Wymeersch, "Position and orientation estimation through millimeter-wave MIMO in 5G systems," IEEE Trans. Wireless Commun., vol. 17, no. 3, pp. 1822-1835, 2018.

[3] L. Liu, C. Oestges, J. Poutanen, K. Haneda, P. Vainikainen, F. Quitin, F. Tufvesson, and P. D. Doncker, "The COST 2100 MIMO channel model," IEEE Wireless Commun., vol. 19, no. 6, pp. 92-99, December 2012.

[4] S.-K. Yong, IEEE Technical Report 15-07-0584-01-003c Std., March 2007.

[5] A. Maltsev, Channel Models for $60 \mathrm{GHz}$ WLAN Systems, IEEE Technical Report 802.11-09/0334r8 Std., May 2008.

[6] Study on channel model for frequencies from 0.5 to $100 \mathrm{GHz}$, 3GPP TR 38.901 version 14.1.1 Std., August 2017.

[7] A. M. Saleh and R. A. Valenzuela, "A statistical model for indoor multipath propagation," IEEE J. Sel. Areas Commun., vol. 5, no. 2, pp. 128-137, Feb. 1987.

[8] A. Maltsev, R. Maslennikov, A. Lomayev, A. Sevastyanov, and A. Khoryaev, "Statistical channel model for $60 \mathrm{GHz}$ WLAN systems in conference room environment," RADIOENGINEERING, vol. 20, no. 2, pp. 409-422, June 2011.

[9] P. Beckmann and A. Spizzichino, "The scattering of electromagnetic waves from rough surfaces," Norwood, MA, Artech House, Inc., 1987, 511 p., 1987.

[10] K. F. Warnick and W. C. Chew, "Numerical simulation methods for rough surface scattering," Waves in random media, vol. 11, no. 1, pp. R1-R30, 2001.

[11] W. S. Ament, "Toward a theory of reflection by a rough surface," Proceedings of the IRE, vol. 41, no. 1, pp. 142-146, Jan 1953.

[12] O. Landron, M. J. Feuerstein, and T. S. Rappaport, "A comparison of theoretical and empirical reflection coefficients for typical exterior wall surfaces in a mobile radio environment," IEEE Trans. Antennas Propag., vol. 44, no. 3, pp. 341-351, Mar 1996.

[13] H. Budiarto, K. Horihata, K. Haneda, and J. Takada, "Superresolution measurement of non-specular wave scattering from building surface roughness," in IEEE 58th Vehicular Technology Conference. IEEE, 2003, pp. 11-15.
[14] V. Degli-Esposti and H. L. Bertoni, "Evaluation of the role of diffuse scattering in urban microcellular propagation," in IEEE 50th Vehicular Technology Conference, vol. 3. IEEE, 1999, pp. 1392-1396.

[15] V. Degli-Esposti, F. Fuschini, E. M. Vitucci, and G. Falciasecca, "Measurement and modelling of scattering from buildings," IEEE Trans. Antennas Propag., vol. 55, no. 1, pp. 143-153, 2007.

[16] V. Degli-Esposti, D. Guiducci, A. de'Marsi, P. Azzi, and F. Fuschini, "An advanced field prediction model including diffuse scattering," IEEE Trans. Antennas Propag., vol. 52, no. 7, pp. 1717-1728, 2004.

[17] F. Mani, F. Quitin, and C. Oestges, "Accuracy of depolarization and delay spread predictions using advanced ray-based modeling in indoor scenarios," EURASIP Journal on wireless communications and networking, vol. 2011, no. 1, p. 11, 2011.

[18] _ - "Directional spreads of dense multipath components in indoor environments: Experimental validation of a ray-tracing approach," IEEE Trans. Antennas Propag., vol. 60, no. 7, pp. 3389-3396, 2012.

[19] J. Järvelainen, K. Haneda, M. Kyro, V.-M. Kolmonen, J.-i. Takada, and $\mathrm{H}$. Hagiwara, "60 GHz radio wave propagation prediction in a hospital environment using an accurate room structural model," in Antennas and Propagation Conference (LAPC), 2012 Loughborough. IEEE, 2012, pp. 1-4.

[20] J. Järveläinen and K. Haneda, "Sixty gigahertz indoor radio wave propagation prediction method based on full scattering model," Radio Science, vol. 49, no. 4, pp. 293-305, 2014.

[21] U. T. Virk, J.-F. Wagen, and K. Haneda, "Simulating specular reflections for point cloud geometrical database of the environment," in Antennas \& Propagation Conference (LAPC), 2015 Loughborough. IEEE, 2015, pp. 1-5.

[22] J.-F. Wagen, U. T. Virk, and K. Haneda, "Measurements based specular reflection formulation for point cloud modelling," in Antennas and Propagation (EuCAP), 2016 10th European Conference on. IEEE, 2016, pp. 1-5.

[23] J. Pascual-García, J. M. Molina-García-Pardo, M. T. MartínezInglés, J. V. Rodríguez, and N. Saurín-Serrano, "On the importance of diffuse scattering model parameterization in indoor wireless channels at mm-wave frequencies," IEEE Access, vol. 4, pp. 688-701, 2016.

[24] L. Tian, V. Degli-Esposti, E. M. Vitucci, X. Yin, F. Mani, and S. X. Lu, "Semi-deterministic modeling of diffuse scattering component based on propagation graph theory," in IEEE 25th Annual International Symposium on Personal, Indoor, and Mobile Radio Communication. IEEE, 2014.

[25] L. Tian, V. Degli-Esposti, E. M. Vitucci, and X. Yin, "Semideterministic radio channel modeling based on graph theory and ray-tracing," IEEE Trans. Antennas Propag., vol. 64, no. 6, pp. 2475-2486, 2016.

[26] F. Fuschini, S. Häfner, M. Zoli, R. Müller, E. M. Vitucci, D. Dupleich, M. Barbiroli, J. Luo, E. Schulz, V. Degli-Esposti et al., "Item level characterization of mm-wave indoor propagation," EURASIP Journal on Wireless Communications and Networking, vol. 2016, no. 1, p. 4, 2016.

[27] E. Leitinger, P. Meissner, C. Ruedisser, G. Dumphart, and K. Witrisal, "Evaluation of position-related information in multipath components for indoor positioning," IEEE J. Sel. Areas Commun., 2015.

[28] K. Witrisal, E. Leitinger, S. Hinteregger, and P. Meissner, "Bandwidth scaling and diversity gain for ranging and positioning in dense multipath channels," IEEE Wireless Commun. Lett., vol. 5, no. 4, pp. 396 - 399, Aug. 2016.

[29] I. S. Merrill, "Radar handbook," John Wiley, 1990.

[30] J. H. Lienhard, A heat transfer textbook. Phlogiston Press, Cambridge, Massachusetts, 2003.

[31] C. Gustafson, $60 \mathrm{GHz}$ Wireless Propagation Channels: Characterization, Modeling and Evaluation, 2014, vol. 69.

[32] C. Gustafson, K. Haneda, S. Wyne, and F. Tufvesson, "On mmwave multipath clustering and channel modeling," IEEE Trans. Antennas Propag., vol. 62, no. 3, pp. 1445-1455, 2014.

[33] P. F. M. Smulders, "Statistical characterization of $60-\mathrm{GHz}$ indoor radio channels," IEEE Trans. Antennas Propag., vol. 57, no. 10, pp. 2820-2829, Oct 2009. 